\title{
FACTOR ANALYSIS: A PROBLEM WITH AGE AND FACTOR DESCRIPTIONS OF THE MOTOR DOMAIN
}

\author{
D. A. DOBBINS
}

\section{University College of Swansea}

Given the increasing recognition that explanations of behavioural domains may well need to be derived from a multivariable data base and recognising the ease of usage of canned statistical computer programmes one statistical technique that involves complex and prolonged computations namely factor analysis is being utilized by behavioural scientists with increasing frequency.

Factor analysis, like any other research tool, if it is to give meaningful results must be applied in an appropriate manner. A subtle yet recognised distortion of simplest factor structure is achieved by factoring a correlation matrix holding spurious correlations. Experimental dependence is most easily observed in an inter-correlation matrix when the total score of two or more variables in the battery selected for factor analysis is added to that battery. Spurious correlations can also arise when the effect of a variable, not a member of the domain selected for factoring, is recognizable across the majority of variables selected for analysis. Such a variable is labelled an 'underlying variable' and is defined as a variable that is (i) not a member of the variable list to be factored and (ii) has significant correlational relation- ships with the majority of variables in the battery selected for factor analysis.

For those analysing a domain or sub-domain that comprises human movement, the problem of the effects of one underlying variable on the resultant factor description needs to be recognized. 'Age' can act as an underlying variable, regardless of the motor ability measured, whether it be of a balance, strength, or finemotor type, children invariably perform at higher levels as they get older.

This maturational effect, easily recognizable via correlations of age with motor performance, causes 'Age', when the range is great enough, to function as an underlying variable.

This paper (i) uses a contrived example to demonstrate the effects of an underlying variable on a factor description (ii) assesses the effect of the underlying variable, 'Age', on a previously reported factor description of a motor domain as substantive and (iii) considers the relevance of factor descriptions when an underlying variable has not been controlled.

\section{SERIAL RESPONSE SPEED IN GAMES PLAYERS}

\author{
R. D. THORPE and J. ATHA \\ Loughborough University of Technology
}

Serial response speed has been examined in all round skilled games players $(n=10)$ and in unskilled subjects $(n=10)$ using two signal serial response tests involving five alternative inter-signal intervals of $100 \mathrm{~ms}$ to 500 ms. Expectancy delays (the delay in the first response) over simple reaction time, because of the expectancy of the second serial signal response events and refractory delays (the delay in the second response) as against simple reaction time because of the occurrence of the preceding serial signal event, were also calculated and examined.
Although the skilled games players had faster simple and serial reaction times than the unskilled $(p<0.05$ ), they did not differ in any important respect from the unskilled, either in expectancy or refractory delays. It is suggested that the advantages of the more rapid reaction time enjoyed by the skilled compared with the unskilled are a linear function of differences in simple reaction time. The possibility of the presence of a post refractory facilitation period was considered. 